\title{
Second harmonic generation with zero phase velocity waves
}

\author{
Walter R. C. Somerville, a) David A. Powell, and Ilya V. Shadrivov \\ Nonlinear Physics Centre, Research School of Physics and Engineering, The Australian National \\ University, Canberra, Australian Capital Territory 0200, Australia
}

(Received 4 March 2011; accepted 30 March 2011; published online 21 April 2011)

\begin{abstract}
We design a dual-band nonlinear composite right-left handed transmission line with phase-matching achieved between the fundamental frequency and second harmonic when both interacting waves have zero phase velocity. Additionally, we show that such a transmission line supports a new regime where the generation of backward second harmonic waves is achieved from a backward fundamental frequency wave. (C) 2011 American Institute of Physics. [doi:10.1063/1.3580616]
\end{abstract}

Composite right-left-handed transmission lines (CRLHTL) are metamaterial-inspired transmission lines, which support both forward and backward propagating modes. ${ }^{1}$ They can be used in applications such as compact resonators, ${ }^{2}$ filters, ${ }^{3}$ and broadband couplers. ${ }^{4}$ The inclusion of nonlinear semiconductor components in the CRLHTL has allowed nonlinear effects to be extensively studied-see Ref. 5 and references therein. In particular, it was shown that the nonlinear CRLHTL can be used for harmonic generation, ${ }^{6}$ as well as for parametric amplification. ${ }^{7}$

Nonlinear processes in media supporting backward (or "left-handed") waves are very unusual. For example, it is possible to generate second harmonics (SHs) in the direction of reflected waves; ${ }^{8}$ this is in a sharp contrast to what happens in typical quadratically nonlinear materials, where the generated SH propagates in the same direction as fundamental frequency (FF). In this letter, we study two new regimes of SH generation. First, we achieve phase matching between two frequencies having zero phase velocity. The regime of zero phase velocity waves is analogous to the epsilon-near zero metamaterial. ${ }^{9}$ We experimentally demonstrate SH generation with both the FF and SH meeting the zero phase velocity condition. Second, we study SH generation for the case when both FF wave and SH are backward waves. We experimentally measure the dispersion properties of the designed transmission lines to confirm that the phase matching conditions are satisfied. This is performed using a highresolution spectral analysis method ${ }^{10,11}$ applied to the measured near-field distribution in the vicinity of the transmission line.

Our design is based on the concept of a balanced CRLHTL, ${ }^{1}$ which has a constant impedance, and continuous dispersion which crosses the zero-phase velocity axis at one frequency with no band gap. ${ }^{1}$ In order to obtain zero phase velocity at the SH frequency while satisfying the balanced condition, we use a dual-band CRLHTL design. ${ }^{12-16}$ We start with the equivalent circuit shown in Fig. 1(a), where we have chosen an asymmetric unit cell to minimize the number of lumped components required. The method for determining the component values for the equivalent circuit was based on the analysis from Ref. 15, modified slightly to take the asymmetry of our circuit into account. ${ }^{12}$

The first constraint is that the unloaded resonant frequency of each LC pair must be identical, $\omega_{o r}^{-2}=L_{h p} C_{h p}$

${ }^{a)}$ Electronic mail: walter.somerville @ vuw.ac.nz.
$=L_{h s} C_{h s}=L_{v p} C_{v p}=L_{v s} C_{v s}$. Since $\beta\left(\omega_{o r}\right)= \pm \pi$, this frequency is at the center of a stop-band separating the first righthanded region from the second left-handed region. The frequencies at which $\beta=0$ are then given by

$$
\begin{aligned}
& \omega_{h z}^{2}=\frac{1}{2}\left\{2 \omega_{o r}^{2}+\omega_{h s h p}^{2} \pm \sqrt{\left(2 \omega_{o r}^{2}+\omega_{h s h p}^{2}\right)^{2}-4 \omega_{o r}^{4}}\right\}, \\
& \omega_{v z}^{2}=\frac{1}{2}\left\{2 \omega_{o r}^{2}+\omega_{v s v p}^{2} \pm \sqrt{\left(2 \omega_{o r}^{2}+\omega_{v s v p}^{2}\right)^{2}-4 \omega_{o r}^{4}}\right\},
\end{aligned}
$$

where $\omega_{h s h p}^{-2}=L_{h s} C_{h p}$ and $\omega_{v s v p}^{-2}=L_{v s} C_{v p}$. In order for the stopbands to be closed, we require that there be only two frequencies where $\beta=0$. This can be achieved by setting $\omega_{h s h p}$ $=\omega_{\text {vsvp }}$, which causes Eqs. (1) and (2) to become equivalent, with roots $\omega_{z 1}^{2}$ and $\omega_{z 2}^{2}$.

We specify that $\beta=0$ at the FF $\omega_{z 1}=2 \pi \times 1 \mathrm{GHz}$ and SH $\omega_{z 2}=2 \pi \times 2 \mathrm{GHz}$. Further constraints are given by the requirement to match the Bloch impedance to the characteristic impedance of our measurement system, $Z_{B} \approx \sqrt{L_{h s} / C_{v p}}$ $=50 \Omega$. Nonlinearity is introduced into the system by imple-

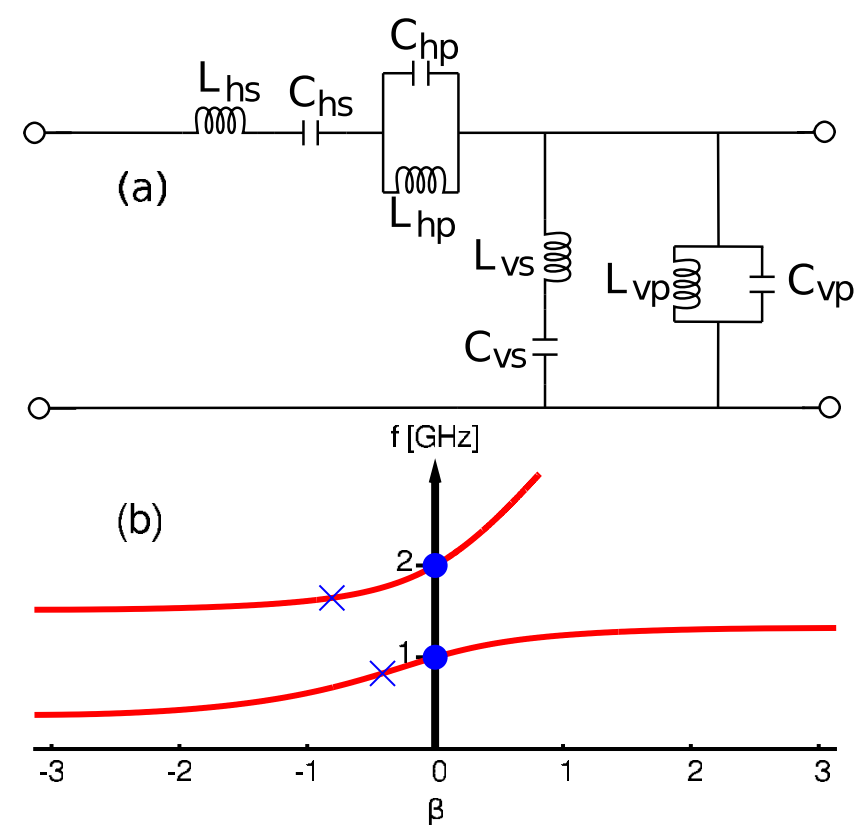

FIG. 1. (Color online) (a) Equivalent circuit of a unit cell of the CRLHTL. (b) Dispersion of the designed balanced transmission line, indicating two backward-wave bands and two forward-wave bands. Circles indicate two zero phase velocity points for which we designed the phase matching. Crosses mark two backward waves for which the phase matching condition is satisfied. 
TABLE I. Parameters of the equivalent circuit model.

\begin{tabular}{lccccccc}
\hline \hline $\begin{array}{c}C_{h s} \\
(\mathrm{pF})\end{array}$ & $\begin{array}{c}C_{h p} \\
(\mathrm{pF})\end{array}$ & $\begin{array}{c}C_{v p} \\
(\mathrm{pF})\end{array}$ & $\begin{array}{c}C_{v s} \\
(\mathrm{pF})\end{array}$ & $\begin{array}{c}L_{h s} \\
(\mathrm{nH})\end{array}$ & $\begin{array}{c}L_{h p} \\
(\mathrm{nH})\end{array}$ & $\begin{array}{c}L_{v s} \\
(\mathrm{nH})\end{array}$ & $\begin{array}{c}L_{v p} \\
(\mathrm{nH})\end{array}$ \\
\hline 2.7 & 5.4 & 1.9 & 0.94 & 4.7 & 2.3 & 13 & 6.8 \\
\hline
\end{tabular}

menting the series capacitances using Skyworks SMV140579LF varactor diodes. This fixes $C_{h s}$ and $C_{h p}$ to be multiples of the diodes' zero bias capacitance of $2.7 \mathrm{pF}$. Under these constraints, we derive the equivalent circuit parameters given in Table I. The linear dispersion of our equivalent circuit model is calculated using the scattering parameters of one unit cell as follows:

$$
\beta(\omega)=\arccos \left(\frac{1-S_{11} S_{22}+S_{21}^{2}}{2 S_{21}}\right) .
$$

The resulting dispersion curves calculated for our structure are shown in Fig. 1(b), demonstrating zero phase velocity points at 1 and $2 \mathrm{GHz}$.

To facilitate experimental realization, we seek to replace most of the components of the equivalent circuit with distributed elements. In order to construct corresponding distributed transmission line components, we use an implementation similar to those presented in Refs. 13, 14, and 16. Our unit cell is depicted in Fig. 2(a), where TL1 replaces $L_{h s}$, TL2 is terminated to ground with a via and replaces $L_{v p}$ and $C_{v p}$, TL4 replaces $L_{v s}$, TL5 replaces $C_{v s}$, and TL3 is introduced to facilitate layout of the circuit. We use $1.6 \mathrm{~mm}$ FR4 as the dielectric substrate, modeled as having a dielectric constant of 4.6. Using the Line Calculation function of the QUCS (Ref. 17) circuit simulation software we find the geometry of the microstrip segments given the characteristic impedance and effective index of a transmission line. Using the $\mathrm{T}$ equivalent circuit $^{18}$ of the transmission line we can relate the characteristic impedance and effective index to the series inductance and shunt capacitance of each line segment.

We iteratively replace the components of the equivalent circuit model with distributed elements. Since each section of inductive transmission line also has some parasitic capacitance, and vice-versa, each replacement introduces unwanted parasitic components into the equivalent circuit model. Therefore we perform a manual adjustment of other circuit parameters to maintain the balanced condition for both crossings. $L_{h p}$ is kept as a lumped element since a microstrip implementation would require very thin track widths.
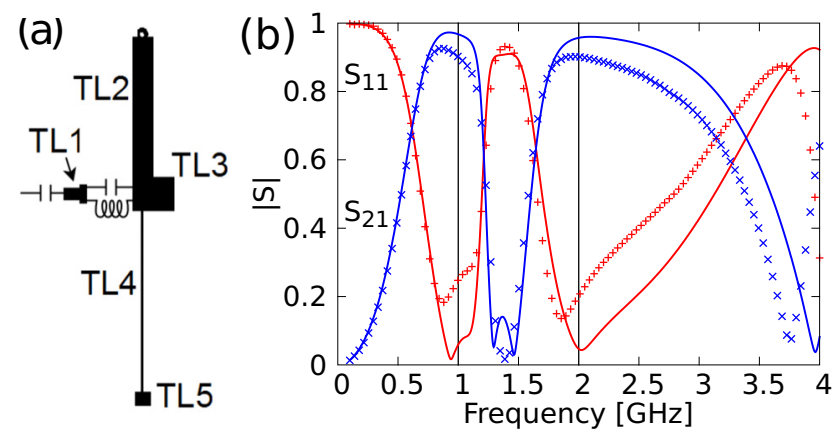

FIG. 2. (Color online) (a) Unit cell of the experimental structure. (b) Comparison between simulated (solid) and measured (dots) $S_{11}$ and $S_{21}$ parameters for a single unit cell of the transmission line.
TABLE II. Dimensions of the transmission line sections.

\begin{tabular}{lrrrrr}
\hline \hline Element & TL1 & TL2 & TL3 & TL4 & TL5 \\
\hline Width (mm) & 1.60 & 2.99 & 4.60 & 0.20 & 2.34 \\
Length (mm) & 3.15 & 19.40 & 2.87 & 25.20 & 1.87 \\
\hline \hline
\end{tabular}

The resulting unit cell structure is then simulated to find its dispersion properties. The conversion from the equivalent circuit to the microstrip implementation results in a shift in the frequencies with zero phase velocity away from those specified. We perform a numerical optimization with the QUCS (Ref. 17) circuit simulator to adjust $L_{h p}$ and the transmission line dimensions to restore the zero phase velocity condition at 1 and $2 \mathrm{GHz}$. We note that this process does not guarantee that the Bloch impedance remains matched. The resulting transmission line dimensions are given in Table II, and $L_{h p}$ was implemented with Tyco Electronics $2.2 \mathrm{nH}$ chip inductors.

We fabricate structures with 1 and 11 unit cells, using photolithography to define the tracks and manually soldering in the lumped components. We perform experimental measurements of S-parameters of a single unit cell using a Rohde and Schwarz ZVB20 vector network analyzer (VNA), and compare these against the S-parameters obtained from simulations. Results are shown in Fig. 2(b) and they demonstrate an excellent agreement between simulated and measured S-parameters. In Fig. 3(a) we show a photograph of the fabricated structure with 11 unit cells.

Next we study the phase-matching required for efficient harmonic generation, based on the experimentally measured dispersion of the transmission line. To measure the dispersion, we use high resolution spectral analysis method, ${ }^{11}$ which analyzes the distribution of the electromagnetic field in the structure. The field distribution is measured using a near-field probe positioned $1 \mathrm{~mm}$ above the transmission line. The near field probe is mounted on an automated translation stage, which allows the amplitude and phase of the

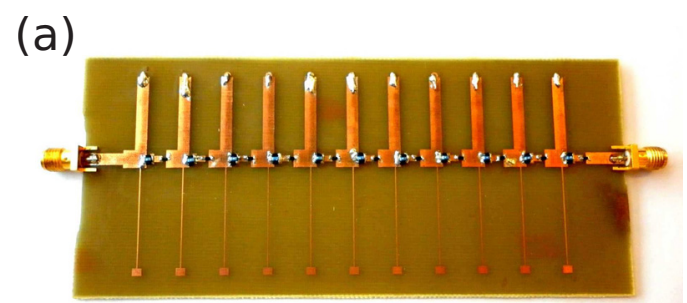

(b) $\begin{array}{lll}0 & 0.2 & 0.4\end{array}$

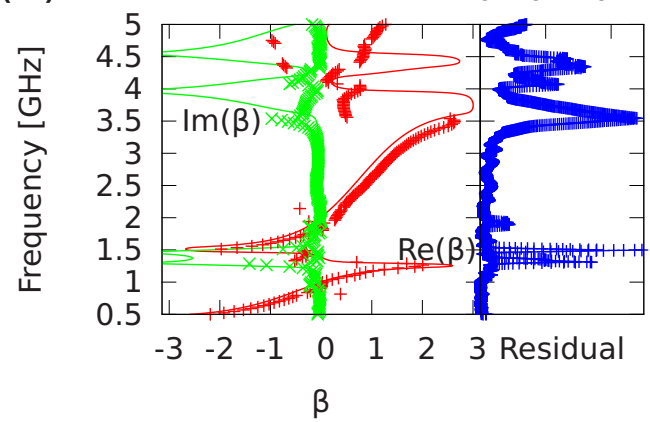

FIG. 3. (Color online) (a) Photograph of the sample containing 11 unit cells (b) Dispersion of the 11-cell structure (points), overlaid with the simulated dispersion (lines). Right plot shows the residual error for dispersion retrieval. 


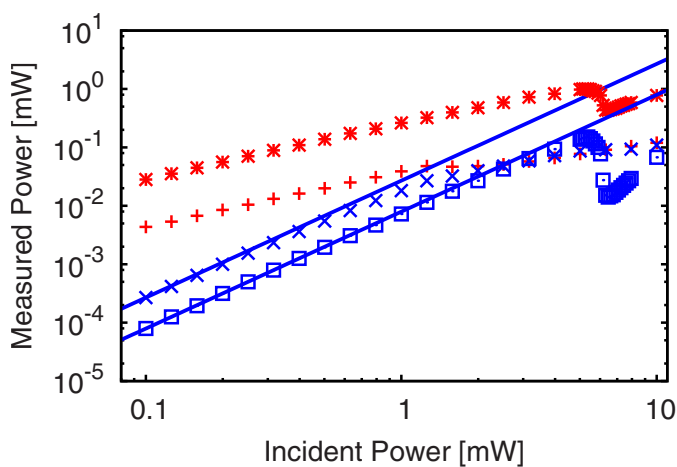

FIG. 4. (Color online) Transmitted FF and SH signals for the case of zero phase velocity phase matching at $1.039 \mathrm{GHz}(+: \mathrm{FF} ; \times$ : SH), and for the backward-to-backward harmonic generation at $0.823 \mathrm{GHz}$, (stars: FF, squares: $\mathrm{SH}$ ). The lines extrapolate the low power $\mathrm{SH}$ signal to show the influence of higher order nonlinear processes.

field distribution along the structure to be measured at all desired frequencies. The transmission line is excited by the VNA and $S_{21}$ measured at each point along the structure is proportional to the local electric field. The dispersion is extracted by fitting forward and backward propagating Bloch modes to the measured data ${ }^{11}$ and is shown in Fig. 3(b).

We compare the experimental results with simulated data and observe excellent agreement. The residual error is also shown, and is quite low at the frequencies of interest, thus giving confidence in the experimentally extracted dispersion curves. Due to manufacturing imperfections and the uncertainty in the dielectric constant of FR4, we observe small band gaps opening near 1 and $2 \mathrm{GHz}$, as indicated by the peaks in the imaginary parts of the wave number $\beta$.

Having determined that the phase matching conditions are well satisfied, we study harmonic generation in our structure. To excite the transmission line, we use a signal generator (Rohde and Schwarz SML-03), and the spectrum of the transmitted signal is measured using a Rohde and Schwarz FSV30 signal analyzer. For fixed input frequency the input power is varied and the power output is recorded at the FF and SH. We observe clear enhancement of the harmonic generation in the two regimes of interest: at $1.039 \mathrm{GHz}$, corresponding to phase matching between waves with zero phase velocity, and at $823 \mathrm{MHz}$, corresponding to phase matching between two backward waves. The results for the transmitted $\mathrm{SH}$ and fundamental frequencies are shown in Fig. 4. At lower power, we observe a clear quadratic dependence of generated SHs on the FF amplitude-as shown by the lines. For larger powers, the generated SH grows more slowly since a significant amount of energy is being transferred to higher order harmonics. Interestingly, at high enough powers for the case of backward-to-backward harmonic generation we observe a sharp drop in both transmitted FF and $\mathrm{SH}$ caused by sharp enhancement in generation of higher-order harmonics.

In conclusion, we designed a transmission line for $\mathrm{SH}$ generation, which allows phase-matching between two zero phase velocity waves, as well as between two backward propagating modes. We fabricated the transmission line, and studied its dispersion properties. We showed experimentally that phase-matching is achieved, both for two zero phase velocity waves, and for two backward waves. This leads to strong $\mathrm{SH}$ generation in the structure.

${ }^{1}$ A. Lai, T. Itoh, and C. Caloz, IEEE Microw. Mag. 5, 34 (2004).

${ }^{2}$ C. Allen, K. Leong, and T. Itoh, Electron. Lett. 42, 96 (2006).

${ }^{3}$ C. Caloz, A. Sanada, and T. Itoh, IEEE Trans. Microwave Theory Tech. 52, 980 (2004).

${ }^{4} \mathrm{M}$. Gil, J. Bonache, J. Garcia-Garcia, J. Martel, and F. Martin, IEEE Trans. Microwave Theory Tech. 55, 1296 (2007)

${ }^{5}$ A. Kozyrev and D. van der Weide, J. Phys. D: Appl. Phys. 41, 173001 (2008).

${ }^{6}$ A. Kozyrev, H. Kim, A. Karbassi, and D. van der Weide, Appl. Phys. Lett. 87, 121109 (2005).

${ }^{7}$ D. Powell, I. Shadrivov, and Y. Kivshar, Appl. Phys. Lett. 94, 084105 (2009).

${ }^{8}$ I. Shadrivov, A. Zharov, and Y. Kivshar, J. Opt. Soc. Am. B 23, 529 (2006).

${ }^{9}$ A. Alù, M. Silveirinha, A. Salandrino, and N. Engheta, Phys. Rev. B 75, 155410 (2007).

${ }^{10}$ A. A. Sukhorukov, S. Ha, I. V. Shadrivov, D.A. Powell, and Y. S. Kivshar, Opt. Express 17, 3716 (2009).

${ }^{11}$ S. Ha, A. A. Sukhorukov, K. B. Dossou, L. C. Botten, C. M. de Sterke, and Y. S. Kivshar, Opt. Lett. 34, 3776 (2009).

${ }^{12}$ A. Rennings, S. Otto, J. Mosig, C. Caloz, and I. Wolff, 2006 Asia-Pacific Microwave Conference (APMC 2006) (December 12-15, Yokohama, Japan, 2006), pp. 1405-1408.

${ }^{13}$ M. Studniberg and G. Eleftheriades, 2007 European Microwave Conference, October 8-12 (Munich, Germany, 2007), p. 408.

${ }^{14}$ M. Studniberg and G. Eleftheriades, IEEE Microw. Wirel. Compon. Lett. 19, 18 (2009).

${ }^{15}$ G. Eleftheriades, IEEE Microw. Wirel. Compon. Lett. 17, 415 (2007).

${ }^{16}$ A. Papanastasiou, G. Georghiou, and G. Eleftheriades, IEEE Microw. Wirel. Compon. Lett. 18, 521 (2008).

${ }^{17}$ Qucs: Quite Universal Circuit Simulator http://qucs.sourceforge.net/.

${ }^{18}$ D. M. Pozar, Microwave Engineering, 3rd ed. (Wiley, New Jersey, 2005). 